\title{
Passive Control of Switched Singular Systems via Output Feedback
}

\author{
Shuhui Shi, ${ }^{1}$ Guoliang Wang, ${ }^{2}$ Jianhua Wang, ${ }^{3}$ and Hong $\mathrm{Li}^{4}$ \\ ${ }^{1}$ Shenyang Institute of Engineering, Shenyang 110136, China \\ ${ }^{2}$ Liaoning Shihua University, Fushun 113001, China \\ ${ }^{3}$ Shenyang University, Shenyang 110004, China \\ ${ }^{4}$ Shenyang Institute of Technology, Fushun 113122, China
}

Correspondence should be addressed to Guoliang Wang; gliangwang@aliyun.com

Received 1 January 2015; Accepted 16 February 2015

Academic Editor: Kun Liu

Copyright (C) 2015 Shuhui Shi et al. This is an open access article distributed under the Creative Commons Attribution License, which permits unrestricted use, distribution, and reproduction in any medium, provided the original work is properly cited.

\begin{abstract}
An instrumental matrix approach to design output feedback passive controller for switched singular systems is proposed in this paper. The nonlinear inequality condition including Lyapunov inverse matrix and controller gain matrix is decoupled by introducing additional instrumental matrix variable. Combined with multiple Lyapunov function method, the nonlinear inequality is transformed into linear matrix inequality (LMI). An LMI condition is presented for switched singular system to be stable and passive via static output feedback under designed switching signal. Moreover, the conditions proposed do not require the decomposition of Lyapunov matrix and its inverse matrix or fixing to a special structure. The theoretical results are verified by means of an example. The method introduced in the paper can be effectively extended to a single singular system and normal switched system.
\end{abstract}

\section{Introduction}

The switched singular systems arise from, for instance, power systems, economic systems, and complex networks. As pointed out in [1], when the interrelationships among different industrial sectors are described and the capital and the demand are variable depending on seasons, the dynamic economic systems are modelled as periodically switched singular systems. In some complex hybrid networks, some algebraic constraints have to be considered. The special algebraic constraints that, for example, communication resources are always limited and required to be allocated to different levels of privileged users, are needed in the resource allocation process. Thus, constructing the network model with a set of constraints is reasonable and indispensable. The model can be denoted by a class of singular hybrid systems [2]. There has been increasing interest in analysis and synthesis for switched singular systems. The stability issues are discussed for continuous-time switched singular systems [3], discrete-time switched singular systems [4], linear switched singular systems $[5,6]$, nonlinear switched singular systems [7], and time-delay switched singular systems [8], respectively. In $[9,10]$, reachability conditions and admissibility criteria are presented, respectively. Reference [11] studies the initial instantaneous jumps at switching points and a sufficient stability condition is obtained for the switched singular system with both stable and unstable subsystems. At arbitrary switching instant, inconsistent state jump for switched singular systems can be compressed by hybrid impulsive controllers in [12]. Filters and observers are designed for switched singular systems in $[13,14]$ and [15], respectively.

It has been shown that passivity is a suitable design approach in power systems [16], neural networks [17], network control [18-20], signal processing [21, 22], Markovian jumping systems [23-25], switched systems [26, 27], and singular systems [28, 29]. In [16], the problem of passive control is considered for uncertain singular time-delay systems, and three types of controllers were designed, namely, state feedback controller, observer-based state feedback controller, and 
dynamic output feedback controller. Under these controllers, the closed-loop systems are quadratically stable and passive. Contingent failures are possible for a real system, which may cause performance of the system to be degraded and even hazard [30]. The work [17] applies passivity-based faulttolerant synchronization control to chaotic neural networks against actuator faults by using the semi-Markov jump model approach. The work [27] investigates the problem of robust reliable passive control for a class of uncertain stochastic switched time-delay systems with actuator failures. Sufficient condition for the stochastic switched time-delay systems to be passive and exponentially stable under switching state feedback controller is derived. The work [29] designs a state feedback controller such that, for all possible actuator failures, the closed-loop singular system is exponentially stable and passive.

Up to now, little attention has been paid to passive control problem for switched singular systems. This motivates us to investigate this problem. Furthermore, considering the operational cost and the reliability of systems and the simplicity of implementation, output feedback is always adopted to stabilize a system. Thus, we study passive control of switched singular systems through output feedback.

In this paper, by introducing instrumental matrix variable, the nonlinear inequality including Lyapunov inverse matrix and controller gain matrix is decoupled, which makes the design of output feedback passive controllers for continuous-time switched singular systems easy. Based on multiple Lyapunov functions and variable substitution techniques, a new and simple sufficient condition is presented in terms of LMI, by solving which static output feedback passive controller can be designed. The novelty of the conditions proposed in this paper lies in the following aspect. Decomposition of Lyapunov matrix and its inverse matrix is not required. Moreover, the Lyapunov inverse matrix is not fixed to a special structure.

The rest of this paper is organized as follows. Problem statement and preliminaries are given in Section 2. Output feedback passive control is studied in Section 3. In Section 4, an example shows the efficiency of main results in the paper. Section 5 concludes this paper.

\section{Problem Statement and Preliminaries}

Consider the following switched singular system:

$$
\begin{gathered}
E_{c i} \dot{x}(t)=A_{c i} x(t)+B_{c i} u(t)+G_{c i} \omega(t) \\
z(t)=H_{c i} x(t)+D_{c i} \omega(t) \\
y(t)=C_{c i} x(t),
\end{gathered}
$$

where $i \in \chi=\{1,2, \ldots, m\}$ is the switching signal. $x(t) \in R^{n}$ is the state vector, $u(t) \in R^{m}$ is the control input vector, $\omega(t) \in L_{2}^{r}[0, \infty)$ is the external disturbance vector, $z(t) \epsilon$ $R^{r}$ is the control input vector, and $y(t) \in R^{p}$ is the measured controlled output vector. $E_{c i}, A_{c i}, B_{c i}, C_{c i}, D_{c i}, G_{c i}, H_{c i}$ are constant matrices with appropriate dimensions. $E_{c i} \in$ $R^{n \times n}$ and rank $E_{c i}=r_{i} \leq n$. Without loss of generality, we assume that $C_{c i}$ is full row rank.
Let us consider the following static output feedback controller:

$$
u(t)=K_{i} y(t)
$$

where $K_{i}$ is the controller gain matrix to be designed.

Then, the resulting closed-loop system can be described as

$$
\begin{gathered}
E_{c i} \dot{x}(t)=\left(A_{c i}+B_{c i} K_{i} C_{c i}\right) x(t)+G_{c i} \omega(t) \\
z(t)=H_{c i} x(t)+D_{c i} \omega(t) \\
y(t)=C_{c i} x(t) .
\end{gathered}
$$

We are now considering the output feedback passive control problem for system (3). In this paper, we aim to design output feedback passive controller such that system (3) simultaneously satisfies the following two requirements.

(i) System (3) with $\omega(t)=0$ is stable.

(ii) For a give scalar $\eta>0$, the dissipation inequality

$$
\int_{0}^{T}\left(\omega^{T} z-\eta \omega^{T} \omega\right) d t \geq 0, \quad \forall T>0,
$$

holds for all trajectories with zero initial condition. In this case, the closed-loop switched singular system (3) is said to be stable and passive with dissipation rate $\eta$.

To obtain the main results of this paper, the following transformation is introduced.

Since $C_{c i}$ is full row rank, there exists a nonsingular matrix $T_{i}$ such that $C_{c i} T_{i}^{-1}=\left[\begin{array}{ll}I_{p} & 0\end{array}\right]$. Using the nonsingular $T_{i}$ as a similarity transformation for system (3), the closed-loop system (3) is equivalent to the following system:

$$
\begin{gathered}
E_{i} \dot{x}(t)=\bar{A}_{i} x(t)+G_{i} \omega(t) \\
z(t)=H_{i} x(t)+D_{i} \omega(t) \\
y(t)=C_{i} x(t),
\end{gathered}
$$

where $E_{i}=T_{i} E_{c i} T_{i}^{-1}, A_{i}=T_{i} A_{c i} T_{i}^{-1}, B_{i}=T_{i} B_{c i}, C_{i}=$ $C_{c i} T_{i}^{-1}=\left[\begin{array}{ll}I_{p} & 0\end{array}\right], G_{i}=T_{i} G_{c i}, H_{i}=H_{c i} T_{i}^{-1}, D_{i}=D_{c i}$, and $\bar{A}_{i}=A_{i}+B_{i} K_{i} C_{i}$.

\section{Output Feedback Passive Control}

The following theorem provides a sufficient condition under which system (5) is stable and passive with dissipation rate $\eta$.

Theorem 1. If there exist simultaneously nonnegative real number $\beta_{i j}, \eta$ and matrices $X_{i}>0, X_{j}>0$ and matrices $K_{i}$ such that for any $i, j \in \chi, i \neq j$,

$$
X_{i}^{T} E_{i}^{T}=E_{i} X_{i} \geq 0
$$


and inequality

$\left[\begin{array}{cc}X_{i}^{T} \bar{A}_{i}^{T}+\bar{A}_{i} X_{i}+\sum_{j=1}^{m} \beta_{i j}\left(E_{i}^{T} X_{i}-E_{j}^{T} X_{j}\right) & G_{i}-X_{i}^{T} H_{i}^{T} \\ G_{i}^{T}-H_{i} X_{i} & 2 \eta I-D_{i}-D_{i}^{T}\end{array}\right]$

$<0$

hold, system (5) is stable and passive with dissipation rate $\eta$ under static output feedback $u(t)=K_{i} y(t)$ via switching signal

$$
i=\arg \max \left\{x^{T}(t) E_{i}^{T} X_{i} x(t), i \in \chi\right\} .
$$

Proof. When $\beta_{i j}$ is simultaneously nonnegative, for $x \in$ $R^{n} /\left\{x \mid \prod_{i=1}^{m} E_{i} x=0\right\}$, there must exist a $i \in \chi=\{1, \ldots, m\}$, such that for any $j \neq i, j \in \chi, x^{T}\left(E_{i}^{T} X_{i}-E_{j}^{T} X_{j}\right) x \geq 0$ holds. Then $\sum_{j=1}^{m} x^{T}\left(E_{i}^{T} X_{i}-E_{j}^{T} X_{j}\right) x \geq 0$ holds. Let

$$
\begin{gathered}
\Omega_{i}=\left\{x \in R^{n} \mid x^{T}\left(E_{i}^{T} X_{i}-E_{j}^{T} X_{j}\right) x \geq 0,\right. \\
\left.\prod_{i=1}^{m} E_{i} x \neq 0, j \neq i, j \in \chi\right\} .
\end{gathered}
$$

Clearly, $\bigcup_{i=1}^{m} \Omega_{i}=R^{n} /\left\{x \mid \prod_{i=1}^{m} E_{i} x=0\right\}$.

Construct $\bar{\Omega}_{1}=\Omega_{1}, \ldots, \bar{\Omega}_{i}=\Omega_{i}-\bigcup_{j=1}^{i-1} \bar{\Omega}_{j}, \ldots, \bar{\Omega}_{m}=$ $\Omega_{m}-\bigcup_{j=1}^{m-1} \bar{\Omega}_{j}$. Obviously, $\bigcup_{i=1}^{m} \bar{\Omega}_{i}=R^{n} /\left\{x \mid \prod_{i=1}^{m} E_{i} x=0\right\}$ and $\bar{\Omega}_{i} \bigcap \bar{\Omega}_{j}=\phi, i \neq j$, hold.

Design switching signal as

$$
i=\arg \max \left\{x^{T}(t) E_{i}^{T} X_{i} x(t), i \in \chi\right\} .
$$

When $x \in \bar{\Omega}_{i}$, choose Lyapunov function as

$$
V(x(t))=x^{T}(t) E_{i}^{T} X_{i}^{-1} x(t) .
$$

Premultiplying $X_{i}^{-T}$ and postmultiplying $X_{i}^{-1}$ to $X_{i}^{T} E_{i}^{T}=$ $E_{i} X_{i}$ in (6), respectively, one gets $E_{i}^{T} X_{i}^{-1}=X_{i}^{-T} E_{i}$. Then, the derivation of Lyapunov function $V(x(t))$ along the closedloop system (5) is

$$
\begin{aligned}
\dot{V}(x(t))= & \dot{x}^{T}(t) E_{i}^{T} X_{i}^{-1} x(t)+x^{T}(t) E_{i}^{T} X_{i}^{-1} \dot{x}(t) \\
= & {\left[\bar{A}_{i} x(t)+G_{i} \omega(t)\right]^{T} X_{i}^{-1} x(t) } \\
& +x^{T}(t) X_{i}^{-T}\left[\bar{A}_{i} x(t)+G_{i} \omega(t)\right] .
\end{aligned}
$$

Therefore

$$
\begin{aligned}
& \dot{V}(x(t))-2 \omega^{T}(t) z(t)+2 \eta \omega^{T}(t) \omega(t) \\
& =\left[\bar{A}_{i} x(t)+G_{i} \omega(t)\right]^{T} X_{i}^{-1} x(t) \\
& +x^{T}(t) X_{i}^{-T}\left[\bar{A}_{i} x(t)+G_{i} \omega(t)\right] \\
& -2 \omega^{T}(t)\left[H_{i} x(t)+D_{i} \omega(t)\right]+2 \eta \omega^{T}(t) \omega(t) \\
& \left.=\left[\begin{array}{c}
x(t) \\
\omega(t)
\end{array}\right]\right]^{T}\left[\begin{array}{cc}
\bar{A}_{i}^{T} X_{i}^{-1}+X_{i}^{-T} \bar{A}_{i} & X_{i}^{-T} G_{i}-H_{i}^{T} \\
G_{i}^{T} X_{i}^{-1}-H_{i} & 2 \eta I-D_{i}-D_{i}^{T}
\end{array}\right]\left[\begin{array}{c}
x(t) \\
\omega(t)
\end{array}\right] \\
& =\zeta^{T}(t) \Xi_{i} \zeta(t),
\end{aligned}
$$

where $\zeta(t)=\left[\begin{array}{c}x(t) \\ \omega(t)\end{array}\right], \Xi_{i}=\left[\begin{array}{cc}\bar{A}_{i}^{T} X_{i}^{-1}+X_{i}^{-T} \bar{A}_{i} & X_{i}^{-T} G_{i}-H_{i}^{T} \\ G_{i}^{T} X_{i}^{-1}-H_{i} & 2 \eta I-D_{i}-D_{i}^{T}\end{array}\right]$. Preand postmultiplying $\Xi_{i}$ by $\left[\begin{array}{cc}X_{i}^{T} & 0 \\ 0 & I\end{array}\right]$ and $\left[\begin{array}{cc}X_{i} & 0 \\ 0 & I\end{array}\right]$, respectively, we obtain

$$
\left[\begin{array}{cc}
X_{i}^{T} & 0 \\
0 & I
\end{array}\right] \Xi_{i}\left[\begin{array}{cc}
X_{i} & 0 \\
0 & I
\end{array}\right]=\Phi_{i}
$$

where $\Phi_{i}=\left[\begin{array}{cc}X_{i}^{T} \bar{A}_{i}^{T}+\bar{A}_{i} X_{i} & G_{i}-X_{i}^{T} H_{i}^{T} \\ G_{i}^{T}-H_{i} X_{i} & 2 \eta I-D_{i}-D_{i}^{T}\end{array}\right]$. Then, $\Xi_{i}<0$ is equivalent to $\Phi_{i}<0$. Next, we prove that $\Phi_{i}<0$ holds.

Suppose that (7) holds. Inequality (7) can be rewritten as

$$
\Phi_{i}+\left[\begin{array}{cc}
\sum_{j=1}^{m} \beta_{i j}\left(E_{i}^{T} X_{i}-E_{j}^{T} X_{j}\right) & 0 \\
0 & 0
\end{array}\right]<0 .
$$

According to switching signal (8), $\Phi_{i}<0$ when (15) holds, and by (13), we get that the following inequality holds:

$$
\dot{V}(x(t))-2 \omega^{T}(t) z(t)+2 \eta \omega^{T}(t) \omega(t) \leq 0 .
$$

Taking the integral on the two sides of (16) from 0 to $T$, we obtain

$$
\int_{0}^{T}\left[\dot{V}(x(t))-2 \omega^{T}(t) z(t)+2 \eta \omega^{T}(t) \omega(t)\right] d t \leq 0 .
$$

To get the result, we introduce

$$
S(T)=\int_{0}^{T}\left[-2 \omega^{T}(t) z(t)+2 \eta \omega^{T}(t) \omega(t)\right] d t,
$$

where $T>0$. Noting the zero initial condition, it can be shown that, for any $T>0$,

$$
S(T) \leq \int_{0}^{T}\left[\dot{V}(x(t))-2 \omega^{T}(t) z(t)+2 \eta \omega^{T}(t) \omega(t)\right] d t .
$$


It follows from (19) that $S(T) \leq 0$, and hence

$$
\int_{0}^{T}\left[-2 \omega^{T}(t) z(t)+2 \eta \omega^{T}(t) \omega(t)\right] d t \leq 0
$$

is satisfied for any $T>0$. The system is passive with dissipation $\eta$.

Next, we prove the stability of system (5) with $\omega(t)=0$. Following the similar procedures as used above, we have

$$
\dot{V}(x(t))=x^{T}(t)\left(\bar{A}_{i}^{T} X_{i}^{-1}+X_{i}^{-T} \bar{A}_{i}\right) x(t) .
$$

Note that (7) implies $\bar{A}_{i}^{T} X_{i}^{-1}+X_{i}^{-T} \bar{A}_{i}<0$. Therefore, we have $\dot{V}(x(t))<0$ when $(7)$ holds. According to Lyapunov theory, system (5) is stable. This completes the proof.

Condition (7) in Theorem 1 is not linear matrix inequality, which cannot be solved by MATLAB. The controller gains $K_{i}$ are also difficult to be computed from these conditions. In order to solve output feedback passive controller, we induce the following important lemma.

Lemma 2. For any $i, j \in \chi$, inequality (7) holds if there exists an instrumental matrix $Y_{i}$ such that

$$
\left[\begin{array}{ccc}
\Psi_{i} & X_{i}^{T}-Y_{i}+\bar{A}_{i} Y_{i}^{T} & G_{i}-X_{i}^{T} H_{i}^{T} \\
X_{i}-Y_{i}^{T}+Y_{i} \bar{A}_{i}^{T} & -Y_{i}-Y_{i}^{T} & 0 \\
G_{i}^{T}-H_{i} X_{i} & 0 & 2 \eta I-D_{i}-D_{i}^{T}
\end{array}\right]<0
$$

where $\Psi_{i}=Y_{i} \bar{A}_{i}^{T}+\bar{A}_{i} Y_{i}^{T}+\sum_{j=1}^{m} \beta_{i j}\left(E_{i}^{T} X_{i}-E_{j}^{T} X_{j}\right)$.

Proof. Let $\Lambda_{i}=\left[\begin{array}{ccc}I & \bar{A}_{i} & 0 \\ 0 & 0 & I\end{array}\right]$; then $\Lambda_{i}$ is full row rank. By setting $\Psi_{i}=Y_{i} \bar{A}_{i}^{T}+\bar{A}_{i} Y_{i}^{T}+\sum_{j=1}^{m} \beta_{i j}\left(E_{i}^{T} X_{i}-E_{j}^{T} X_{j}\right)$, the result of Lemma 2 can be evidently derived from the fact that

$$
\begin{gathered}
\Lambda_{i} \cdot\left[\begin{array}{ccc}
\Psi_{i} & X_{i}^{T}-Y_{i}+\bar{A}_{i} Y_{i}^{T} & G_{i}-X_{i}^{T} H_{i}^{T} \\
X_{i}-Y_{i}^{T}+Y_{i} \bar{A}_{i}^{T} & -Y_{i}-Y_{i}^{T} & 0 \\
G_{i}^{T}-H_{i} X_{i} & 0 & 2 \eta I-D_{i}-D_{i}^{T}
\end{array}\right] \cdot \Lambda_{i}^{T} \\
=\left[\begin{array}{cc}
X_{i}^{T} \bar{A}_{i}^{T}+\bar{A}_{i} X_{i}+\sum_{j=1}^{m} \beta_{i j}\left(E_{i}^{T} X_{i}-E_{j}^{T} X_{j}\right) & G_{i}-X_{i}^{T} H_{i}^{T} \\
G_{i}^{T}-H_{i} X_{i} & 2 \eta I-D_{i}-D_{i}^{T}
\end{array}\right] .
\end{gathered}
$$

Remark 3. The instrumental matrix variable $Y_{i}$ is introduced to decouple Lyapunov inverse matrix $X_{i}$ and controller gain matrix $K_{i}$, which makes the design of output feedback passive controllers feasible, and, at the same time, $X_{i}$ and $X_{i}^{-1}$ do not require decomposition or fixing to a certain structure.

Based on the above lemma, an LMI condition is presented, under which system (5) is stable and passive in the following theorem.
Theorem 4. If there exist simultaneously nonnegative real number $\beta_{i j}, \eta$ and matrices $Z_{i}>0, Z_{j}>0, Y_{i}, W_{i}, U_{i}, U_{j}$ such that for any $i, j \in \chi, i \neq j$,

$$
\left[\begin{array}{ccc}
\Theta_{i 11} & \Theta_{i 12} & \Theta_{i 13} \\
\Theta_{i 12}^{T} & -Y_{i}-Y_{i}^{T} & 0 \\
\Theta_{i 13}^{T} & 0 & 2 \eta I-D_{i}-D_{i}^{T}
\end{array}\right]<0
$$

where $\Theta_{i 11}=Y_{i} A_{i}^{T}+A_{i} Y_{i}^{T}+B_{i} W_{i}+W_{i}^{T} B_{i}^{T}+\sum_{j=1}^{m} \beta_{i j}\left(E_{i}^{T} Z_{i} E_{i}^{T}+\right.$ $\left.E_{i}^{T} L_{i} U_{i}-E_{j}^{T} Z_{j} E_{j}^{T}-E_{j}^{T} L_{j} U_{j}\right), \Theta_{i 12}=E_{i} Z_{i}^{T}+U_{i}^{T} L_{i}^{T}-Y_{i}+$ $A_{i} Y_{i}^{T}+B_{i} W_{i}, \Theta_{i 13}=G_{i}-E_{i} Z_{i}^{T} H_{i}^{T}-U_{i}^{T} L_{i}^{T} H_{i}^{T}, Y_{i}=\left[\begin{array}{cc}Y_{i 11} & Y_{i 12} \\ 0 & Y_{i 22}\end{array}\right]$, and $W_{i}=\left[\begin{array}{ll}W_{i 1} & 0\end{array}\right]$, then, system (5) is stable and passive with dissipation rate $\eta$ under static output feedback

$$
K_{i}=W_{i 1} Y_{i 11}^{-T}
$$

via switching signal

$$
i=\arg \max \left\{x^{T}(t) E_{i}^{T}\left(Z_{i} E_{i}^{T}+L_{i} U_{i}\right) x(t), i \in \chi\right\} .
$$

Proof. Since $C_{i}=\left[\begin{array}{ll}I_{p} & 0\end{array}\right]$ in $\bar{A}_{i}=A_{i}+B_{i} K_{i} C_{i}$, then $B_{i} K_{i} C_{i} Y_{i}^{T}=$ $B_{i}\left[\begin{array}{ll}K_{i} & 0\end{array}\right] Y_{i}^{T}$. Letting $Y_{i}=\left[\begin{array}{cc}Y_{i 11} & Y_{i 12} \\ 0 & Y_{i 22}\end{array}\right]$, one gets $Y_{i}^{T}=\left[\begin{array}{cc}Y_{i 11}^{T} & 0 \\ Y_{i 12}^{T} & Y_{i 22}^{T}\end{array}\right]$. Thus, $B_{i}\left[\begin{array}{ll}K_{i} & 0\end{array}\right] Y_{i}^{T}=B_{i}\left[\begin{array}{ll}K_{i} Y_{i 11}^{T} & 0\end{array}\right]$. Let $W_{i}=\left[\begin{array}{ll}W_{i 1} & 0\end{array}\right], W_{i 1}=$ $K_{i} Y_{i 11}^{T}$. A static output feedback gain is denoted by $K_{i}=$ $W_{i 1} Y_{i 11}^{-T}$.

By making use of the existent methods (e.g., [31, 32]), we introduce the matrix $L_{i} \in R^{n \times\left(n-r_{i}\right)}$ with $\operatorname{rank} L_{i}=n-r_{i}$ satisfying $E_{i} L_{i}=0$. By setting $X_{i}=Z_{i} E_{i}^{T}+L_{i} U_{i}$, one obtains that $X_{i}^{-T} E_{i}^{T}=E_{i} X_{i} \geq 0$ holds when $Z_{i}>0$. Then, according to Lemma 2 , inequality (6) and (7) can be rewritten as inequality (24). Inequality (24) is a linear matrix inequality. This completes the proof.

Remark 5. If $\beta_{i j}$ is nonpositive simultaneously, Theorem 4 also holds through choosing the switching signal as $i=$ $\arg \min \left\{x^{T}(t) E_{i}^{T}\left(Z_{i} E_{i}^{T}+L_{i} U_{i}\right) x(t), i \in \chi\right\}$.

Remark 6. When switched singular system reduces to a single singular system (i.e., no switching), Theorem 4 also holds by setting $A_{i}=A, B_{i}=B, G_{i}=G$, and so forth. This will be illustrated in Corollary 7. When switched singular system reduces to a normal switched system (i.e., $E_{c i}=I$ ), the method introduced in this paper is also available, and, at the same time, Theorem 4 is rewritten as Corollary 8 .

Corollary 7. If there exist a real number $\eta>0$ and matrices $Z>0, Y, W, U$ such that

$$
\left[\begin{array}{ccc}
\Theta_{11} & \Theta_{12} & \Theta_{13} \\
\Theta_{12}^{T} & -Y-Y^{T} & 0 \\
\Theta_{13}^{T} & 0 & 2 \eta I-D-D^{T}
\end{array}\right]<0
$$

where $\Theta_{11}=Y A^{T}+A Y^{T}+B W+W^{T} B^{T}, \Theta_{12}=E Z^{T}+U^{T} L^{T}-$ $Y+A Y^{T}+B W, \Theta_{13}=G-E Z^{T} H^{T}-U^{T} L^{T} H^{T}, Y=\left[\begin{array}{cc}Y_{11} & Y_{12} \\ 0 & Y_{22}\end{array}\right]$, and $W=\left[\begin{array}{ll}W_{1} & 0\end{array}\right]$, then, the corresponding singular system is 
stable and passive with dissipation rate $\eta$ under static output feedback

$$
K=W_{1} Y_{11}^{-T}
$$

Corollary 8. If there exist simultaneously nonnegative real number $\beta_{i j}, \eta$ and matrices $X_{i}>0, X_{j}>0, Y_{i}, W_{i}$ such that for any $i, j \in \chi, i \neq j$,

$$
\left[\begin{array}{ccc}
\Theta_{i 11} & \Theta_{i 12} & \Theta_{i 13} \\
\Theta_{i 12}^{T} & -Y_{i}-Y_{i}^{T} & 0 \\
\Theta_{i 13}^{T} & 0 & 2 \eta I-D_{i}-D_{i}^{T}
\end{array}\right]<0,
$$

where $\Theta_{i 11}=Y_{i} A_{i}^{T}+A_{i} Y_{i}^{T}+B_{i} W_{i}+W_{i}^{T} B_{i}^{T}+\sum_{j=1}^{m} \beta_{i j}\left(X_{i}-X_{j}\right)$, $\Theta_{i 12}=X_{i}^{T}-Y_{i}+A_{i} Y_{i}^{T}+B_{i} W_{i}, \Theta_{i 13}=G_{i}-X_{i}^{T} H_{i}^{T}, Y_{i}=$ $\left[\begin{array}{cc}Y_{i 11} & Y_{i 12} \\ 0 & Y_{i 22}\end{array}\right]$, and $W_{i}=\left[\begin{array}{ll}W_{i 1} & 0\end{array}\right]$, then, the corresponding switched system is stable and passive with dissipation rate $\eta$ under static output feedback

$$
K_{i}=W_{i 1} Y_{i 11}^{-T}
$$

via switching signal

$$
i=\arg \max \left\{x^{T}(t) X_{i} x(t), i \in \chi\right\} .
$$

\section{Example}

Consider the switched singular system composed of two subsystems

$$
\begin{gathered}
E_{c i} \dot{x}(t)=A_{c i} x(t)+B_{c i} u(t)+G_{c i} \omega(t) \\
z(t)=H_{c i} x(t)+D_{c i} \omega(t) \\
y(t)=C_{c i} x(t) \\
i=1,2,
\end{gathered}
$$

where

$$
\begin{aligned}
E_{c 1}=\left[\begin{array}{ll}
1 & 0 \\
0 & 0
\end{array}\right], & E_{c 2}=\left[\begin{array}{ll}
1 & 0 \\
0 & 0
\end{array}\right], \\
A_{c 1}=\left[\begin{array}{cc}
5 & -2 \\
10 & -15
\end{array}\right], & A_{c 2}=\left[\begin{array}{ll}
-6 & -1 \\
-1 & 1.5
\end{array}\right], \\
B_{c 1}=\left[\begin{array}{l}
-3 \\
2.5
\end{array}\right], & B_{c 2}=\left[\begin{array}{l}
-4 \\
2
\end{array}\right], \\
C_{c 1}=\left[\begin{array}{ll}
2 & 1
\end{array}\right], & C_{c 2}=\left[\begin{array}{ll}
-1 & 2
\end{array}\right], \\
G_{c 1}=\left[\begin{array}{l}
0 \\
1
\end{array}\right], & G_{c 2}=\left[\begin{array}{l}
0 \\
1
\end{array}\right], \\
D_{c 1}=2, & D_{c 2}=1, \\
H_{c 1}=\left[\begin{array}{ll}
1 & 1
\end{array}\right], & H_{c 2}=\left[\begin{array}{ll}
1 & 2
\end{array}\right] .
\end{aligned}
$$

Choose similarity transformation matrices as

$$
T_{1}=\left[\begin{array}{cc}
2 & 1 \\
-1 & -1
\end{array}\right], \quad T_{2}=\left[\begin{array}{cc}
-1 & 2 \\
1 & -1
\end{array}\right] .
$$

Then, we get an equivalent state-space description of the above system:

$$
\begin{gathered}
E_{i} \dot{x}(t)=A_{i} x(t)+B_{i} u(t)+G_{i} \omega(t) \\
z(t)=H_{i} x(t)+D_{i} \omega(t) \\
y(t)=C_{i} x(t), \\
i=1,2,
\end{gathered}
$$

where

$$
\begin{aligned}
& E_{1}=T_{1} E_{c 1} T_{1}^{-1}=\left[\begin{array}{cc}
2 & 2 \\
-1 & -1
\end{array}\right], \\
& E_{2}=T_{2} E_{c 2} T_{2}^{-1}=\left[\begin{array}{cc}
-1 & -2 \\
1 & 2
\end{array}\right],
\end{aligned}
$$$$
A_{1}=T_{1} A_{c 1} T_{1}^{-1}=\left[\begin{array}{cc}
39 & 58 \\
-32 & -49
\end{array}\right] \text {, }
$$$$
A_{2}=T_{2} A_{c 2} T_{2}^{-1}=\left[\begin{array}{cc}
8 & 12 \\
-7.5 & -12.5
\end{array}\right] \text {, }
$$$$
B_{1}=T_{1} B_{c 1}=\left[\begin{array}{c}
-3.5 \\
0.5
\end{array}\right] \text {, }
$$$$
B_{2}=T_{2} B_{c 2}=\left[\begin{array}{c}
8 \\
-6
\end{array}\right] \text {, }
$$$$
C_{1}=C_{c 1} T_{1}^{-1}=\left[\begin{array}{l}
1 \\
0
\end{array}\right]^{T},
$$

$$
C_{2}=C_{c 2} T_{2}^{-1}=\left[\begin{array}{l}
1 \\
0
\end{array}\right]^{T}
$$

$D_{1}=D_{c 1}=2$,

$D_{2}=D_{c 2}=1$,

$G_{1}=T_{1} G_{c 1}=\left[\begin{array}{c}1 \\ -1\end{array}\right]$,

$G_{2}=T_{2} G_{c 2}=\left[\begin{array}{c}2 \\ -1\end{array}\right]$

$H_{1}=H_{c 1} T_{1}^{-1}=\left[\begin{array}{ll}0 & -1\end{array}\right]$,

$H_{2}=H_{c 2} T_{2}^{-1}=\left[\begin{array}{ll}3 & 4\end{array}\right]$. 
Choose $\beta_{12}=\beta_{21}=0.5, L_{1}=\left[\begin{array}{c}1 \\ -1\end{array}\right], L_{2}=\left[\begin{array}{c}-2 \\ 1\end{array}\right]$. By solving linear matrix inequality (24) in Theorem 4 , we can obtain

$$
\begin{aligned}
Z_{1} & =\left[\begin{array}{ll}
6.1107 & 1.6528 \\
1.6528 & 0.9175
\end{array}\right], \\
Z_{2} & =\left[\begin{array}{cc}
2.0404 & -1.1790 \\
-1.1790 & 0.9889
\end{array}\right], \\
Y_{1} & =\left[\begin{array}{cc}
0.6280 & -0.0791 \\
0 & 0.0364
\end{array}\right], \\
Y_{2} & =\left[\begin{array}{cc}
2.7362 & -1.3902 \\
0 & 0.5073
\end{array}\right], \\
W_{1} & =\left[\begin{array}{ll}
10.0425 & 0
\end{array}\right] \\
W_{2} & =\left[\begin{array}{ll}
-2.2475 & 0
\end{array}\right] \\
X_{1} & =\left[\begin{array}{cc}
19.9846 & -6.8114 \\
0.6831 & -3.5225
\end{array}\right], \\
X_{2}^{-1} & =\left[\begin{array}{ll}
0.8729 & 1.2647 \\
1.6543 & 2.8273
\end{array}\right] \\
X_{2} & =\left[\begin{array}{cc}
7.5201 & -3.3638 \\
-4.4000 & 2.3218
\end{array}\right], \\
X_{1}^{-1} & =\left[\begin{array}{ll}
0.0536 & -0.1036 \\
0.0104 & -0.3040
\end{array}\right],
\end{aligned}
$$

By linear matrix inequality (24), output feedback passive control gains can be obtained:

$$
K_{1}=15.9905, \quad K_{2}=-0.8214 .
$$

The dissipation rate can be obtained:

$$
\eta=0.5
$$

The corresponding switching signal is chosen as

$$
\begin{gathered}
i= \begin{cases}1, & x(t) \in \Omega_{1} \\
2, & x(t) \in \frac{\Omega_{2}}{\Omega_{1}},\end{cases} \\
\Omega_{1}=\left\{x \in R^{n} \mid x^{T}\left(E_{1}^{T} X_{1}-E_{2}^{T} X_{2}\right) x \geq 0,\right. \\
\left.E_{1} x \neq 0, E_{2} x \neq 0\right\} \\
\Omega_{2}=\left\{x \in R^{n} \mid x^{T}\left(E_{2}^{T} X_{2}-E_{1}^{T} X_{1}\right) x \geq 0,\right. \\
\left.E_{1} x \neq 0, E_{2} x \neq 0\right\} .
\end{gathered}
$$

Under the switching signal, the response curve of the above system is exhibited in Figure 1, where $\omega(t)=\sin t e^{-0.1 t}$. From Figure 1, it is obvious that the resulting closed-loop system is stable.

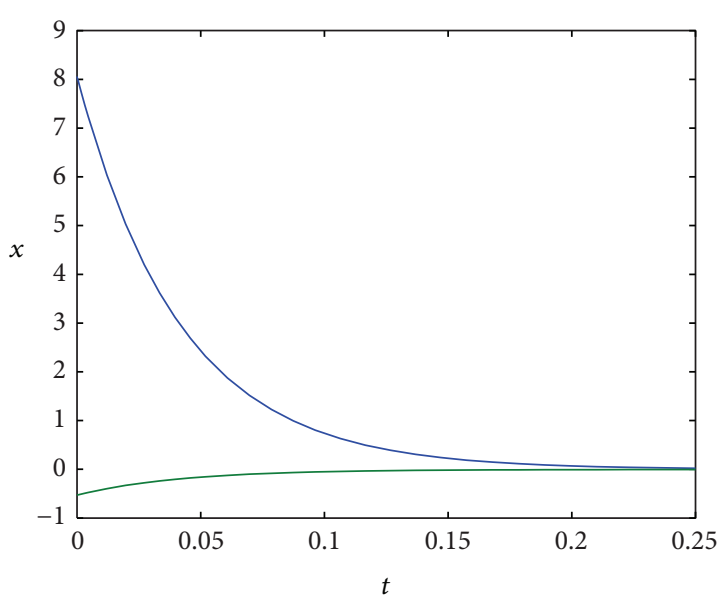

FIGURE 1: State response of the corresponding closed-loop system.

\section{Conclusion}

In this paper, the output feedback passive control problem for a class of switched singular systems is investigated. A novel method is proposed to solve static output feedback passive controllers. Sufficient linear matrix inequality condition is presented by means of introducing instrumental matrix variable $Y_{i}$ and multiple Lyapunov function technique under designed switching law. An example is given to verify the LMI condition proposed for the resulting closed-loop system to be stable and passive with a lower dissipation rate $\eta$. Passivity is a suitable design approach in network control. How to extend the results of this paper to network-based control is an interesting problem. This problem deserves a further study and it remains as our future work.

\section{Conflict of Interests}

The authors declare that there is no conflict of interests regarding the publication of this paper.

\section{Acknowledgments}

This work is supported by the National Natural Science Foundation of China under Grants nos. 61203001, 61104066, and 61473140, Liaoning Educational Committee Foundation under Contract L2014525, and the Natural Science Foundation of Liaoning Province under Grant no. 2014020106.

\section{References}

[1] B. Cantó, C. Coll, and E. Sánchez, "Positive N-periodic descriptor control systems," Systems \& Control Letters, vol. 53, no. 5, pp. 407-414, 2004.

[2] W. Xiong, D. W. C. Ho, and J. Cao, "Synchronization analysis of singular hybrid coupled networks," Physics Letters, Section A: General, Atomic and Solid State Physics, vol. 372, no. 44, pp. 6633-6637, 2008. 
[3] Y. Q. Xia, E.-K. Boukas, P. Shi, and J. H. Zhang, "Stability and stabilization of continuous-time singular hybrid systems," Automatica, vol. 45, no. 6, pp. 1504-1509, 2009.

[4] G. S. Zhai, X. P. Xu, and D. W. C. Ho, "Stability of switched linear discrete-time descriptor systems: a new commutation condition," International Journal of Control, vol. 85, no. 11, pp. 1779-1788, 2012.

[5] D. Liberzon and S. Trenn, "On stability of linear switched differential algebraic equations," in Proceedings of the 48th IEEE Conference on Decision and Control (CDC '09), pp. 2156-2161, December 2009.

[6] J. Clotet, J. Ferrer, and M. D. Magret, "Switched singular linear systems," in Proceedings of the 17th Mediterranean Conference on Control and Automation (MED '09), pp. 1343-1347, Thessaloniki, Greece, June 2009.

[7] F. Long, Y. Xiao, X. Chen, and Z. H. Zhang, "Disturbance attenuation for nonlinear switched descriptor systems based on neural network," Neural Computing and Applications, vol. 23, no. 7-8, pp. 2211-2219, 2013.

[8] I. Zamani and M. Shafiee, "On the stability issues of switched singular time-delay systems with slow switching based on average dwell-time," International Journal of Robust and Nonlinear Control, vol. 24, no. 4, pp. 595-624, 2014.

[9] B. Meng and J.-F. Zhang, "Reachability conditions for switched linear singular systems," IEEE Transactions on Automatic Control, vol. 51, no. 3, pp. 482-488, 2006.

[10] X. Y. Ding, X. Liu, and S. M. Zhong, "Delay-independent criteria for exponential admissibility of switched descriptor delayed systems," Applied Mathematics and Computation, vol. 228, pp. 432-445, 2014.

[11] L. Zhou, D. W. Ho, and G. Zhai, "Stability analysis of switched linear singular systems," Automatica, vol. 49, no. 5, pp. 14811487, 2013.

[12] S. H. Shi, Q. L. Zhang, Z. H. Yuan, and W. Q. Liu, "Hybrid impulsive control for switched singular systems," IET Control Theory \& Applications, vol. 5, no. 1, pp. 103-111, 2011.

[13] J. X. Lin, S. M. Fei, and Q. Wu, "Reliable $H_{\infty}$ filtering for discrete-time switched singular systems with time-varying delay," Circuits, Systems, and Signal Processing, vol. 31, no. 3, pp. 1191-1214, 2012.

[14] D. Zhang, L. Yu, Q.-G. Wang, C.-J. Ong, and Z.-G. Wu, "Exponential $H_{\infty}$ filtering for discrete-time switched singular systems with time-varying delays," Journal of the Franklin Institute, vol. 349, no. 7, pp. 2323-2342, 2012.

[15] D. Koenig, B. Marx, and D. Jacquet, "Unknown input observers for switched nonlinear discrete time descriptor systems," IEEE Transactions on Automatic Control, vol. 53, no. 1, pp. 373-379, 2008.

[16] Q. Li, Q. L. Zhang, N. Yi, and Y. H. Yuan, "Robust passive control for uncertain time-delay singular systems," IEEE Transactions on Circuits and Systems I: Regular Papers, vol. 56, no. 3, pp. 653663, 2009.

[17] J. Wang and H. Shen, "Passivity-based fault-tolerant synchronization control of chaotic neural networks against actuator faults using the semi-Markov jump model approach," Neurocomputing, vol. 143, pp. 51-56, 2014.

[18] H. J. Gao, T. W. Chen, and T. Y. Chai, "Passivity and passification for networked control systems," SIAM Journal on Control and Optimization, vol. 46, no. 4, pp. 1299-1322, 2007.

[19] K. Liu and E. Fridman, "Wirtinger's inequality and Lyapunovbased sampled-data stabilization," Automatica, vol. 48, no. 1, pp. 102-108, 2012.
[20] K. Liu, E. Fridman, and L. Hetel, "Stability and $L_{2}$-gain analysis of networked control systems under Round-Robin scheduling: a time-delay approach," Systems \& Control Letters, vol. 61, pp. 666-675, 2012.

[21] Z. Feng and J. Lam, "Robust reliable dissipative filtering for discrete delay singular systems," Signal Processing, vol. 92, no. 12, pp. 3010-3025, 2012.

[22] Z.-G. Wu, J. H. Park, H. Su, B. Song, and J. Chu, "Mixed $H_{\infty}$ and passive filtering for singular systems with time delays," Signal Processing, vol. 93, no. 7, pp. 1705-1711, 2013.

[23] Z. L. Xia, J. M. Li, and J. R. Li, "Passivity-based resilient adaptive control for fuzzy stochastic delay systems with Markovian switching," Journal of the Franklin Institute, vol. 351, no. 7, pp. 3818-3836, 2014.

[24] S. He and F. Liu, "Optimal finite-time passive controller design for uncertain nonlinear Markovian jumping systems," Journal of the Franklin Institute: Engineering and Applied Mathematics, vol. 351, no. 7, pp. 3782-3796, 2014.

[25] X. M. Yao and L. Guo, "Composite disturbance-observer-based output feedback control and passive control for Markovian jump systems with multiple disturbances," IET Control Theory \& Applications, vol. 8, no. 10, pp. 873-881, 2014.

[26] J. C. Geromel, P. Colaneri, and P. Bolzern, "Passivity of switched linear systems: analysis and control design," Systems and Control Letters, vol. 61, no. 4, pp. 549-554, 2012.

[27] H. M. Jia, Z. R. Xiang, and H. R. Karimi, "Robust reliable passive control of uncertain stochastic switched time-delay systems," Applied Mathematics and Computation, vol. 231, pp. 254-267, 2014.

[28] G. L. Wang, Q. L. Zhang, and C. Y. Yang, "Dissipative control for singular Markovian jump systems with time delay," Optimal Control Applications \& Methods, vol. 33, no. 4, pp. 415-432, 2012.

[29] Z.-G. Wu, J. H. Park, H. Su, and J. Chu, "Reliable passive control for singular systems with time-varying delays," Journal of Process Control, vol. 23, no. 8, pp. 1217-1228, 2013.

[30] H. Y. Li, H. J. Gao, P. Shi, and X. D. Zhao, "Fault-tolerant control of Markovian jump stochastic systems via the augmented sliding mode observer approach," Automatica, vol. 50, no. 7, pp. 1825-1834, 2014.

[31] L. Q. Zhang, B. Huang, and J. Lam, "LMI synthesis of $H_{2}$ and mixed $\mathrm{H}_{2} / \mathrm{H}_{\infty}$ controllers for singular systems," IEEE Transactions on Circuits and Systems II: Analog and Digital Signal Processing, vol. 50, no. 9, pp. 615-626, 2003.

[32] E. Uezato and M. Ikeda, "Strict LMI conditions for stability, robust stabilization, and $H_{\infty}$ control of descriptor systems," in Proceedings of the 38th IEEE Conference on Decision and Control (CDC '99), vol. 4, pp. 4092-4097, IEEE, Phoenix, Ariz, USA, December 1999. 


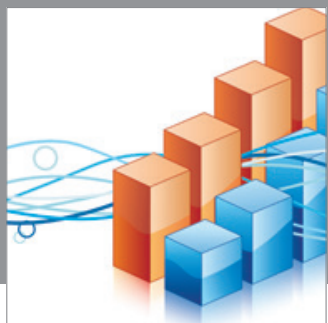

Advances in

Operations Research

mansans

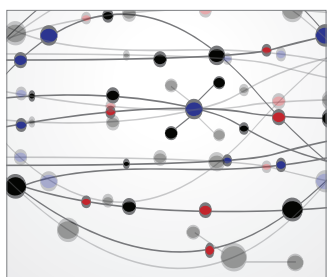

The Scientific World Journal
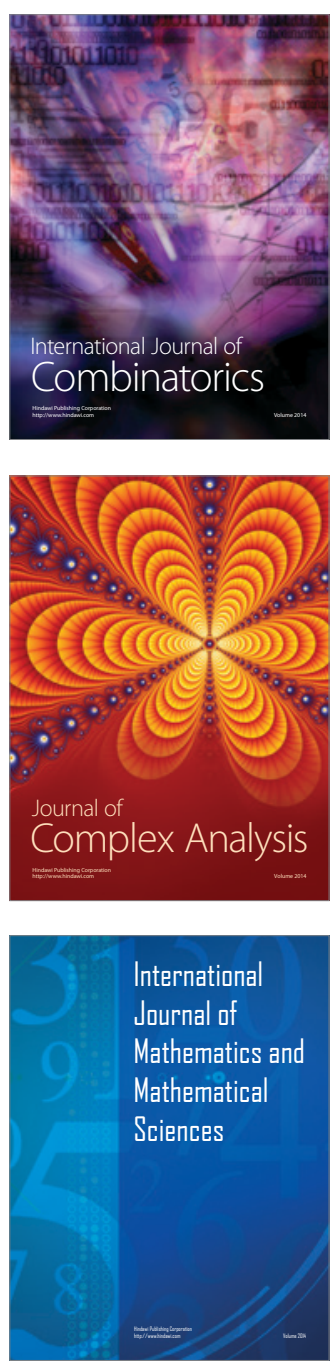
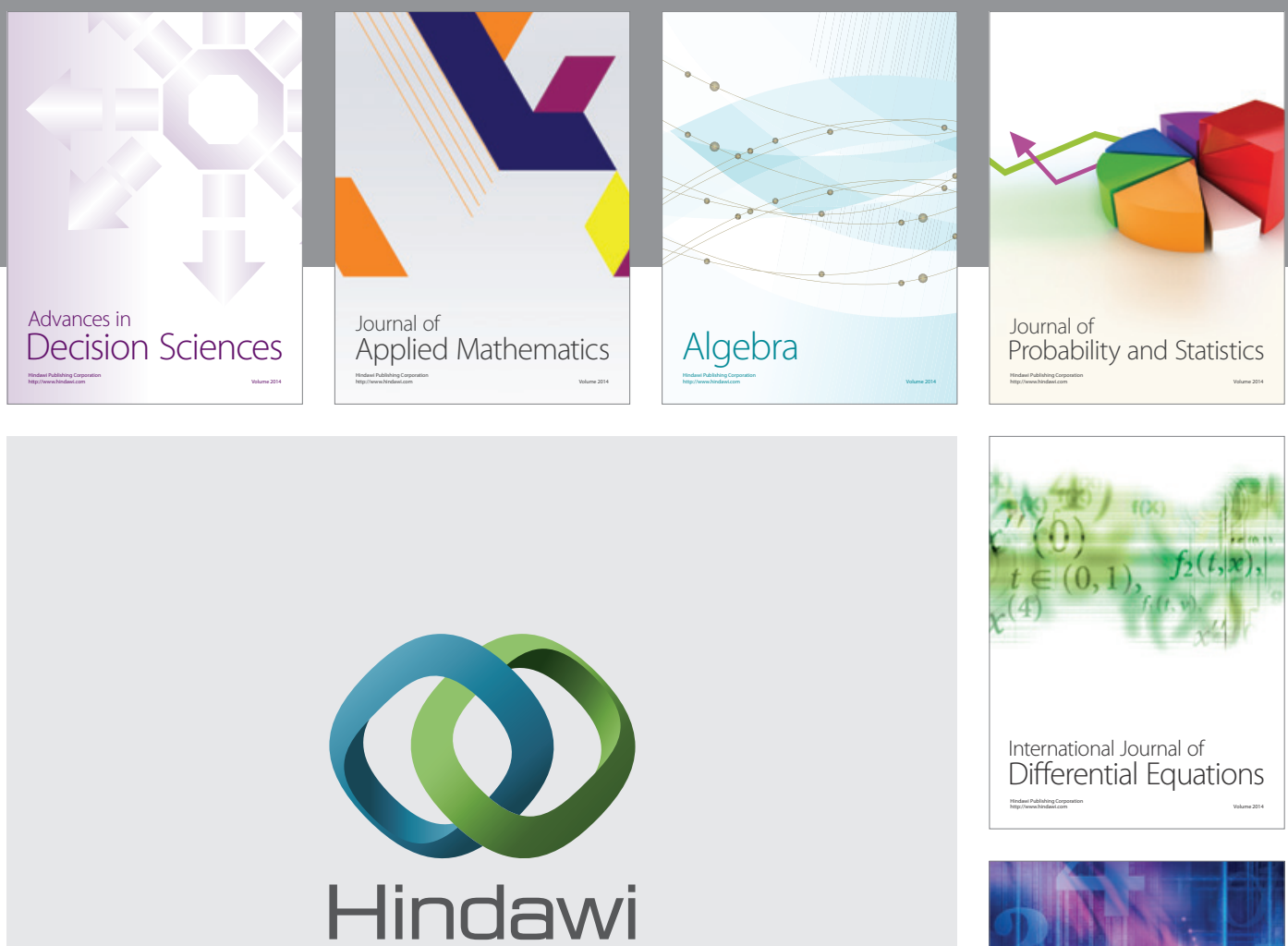

Submit your manuscripts at http://www.hindawi.com
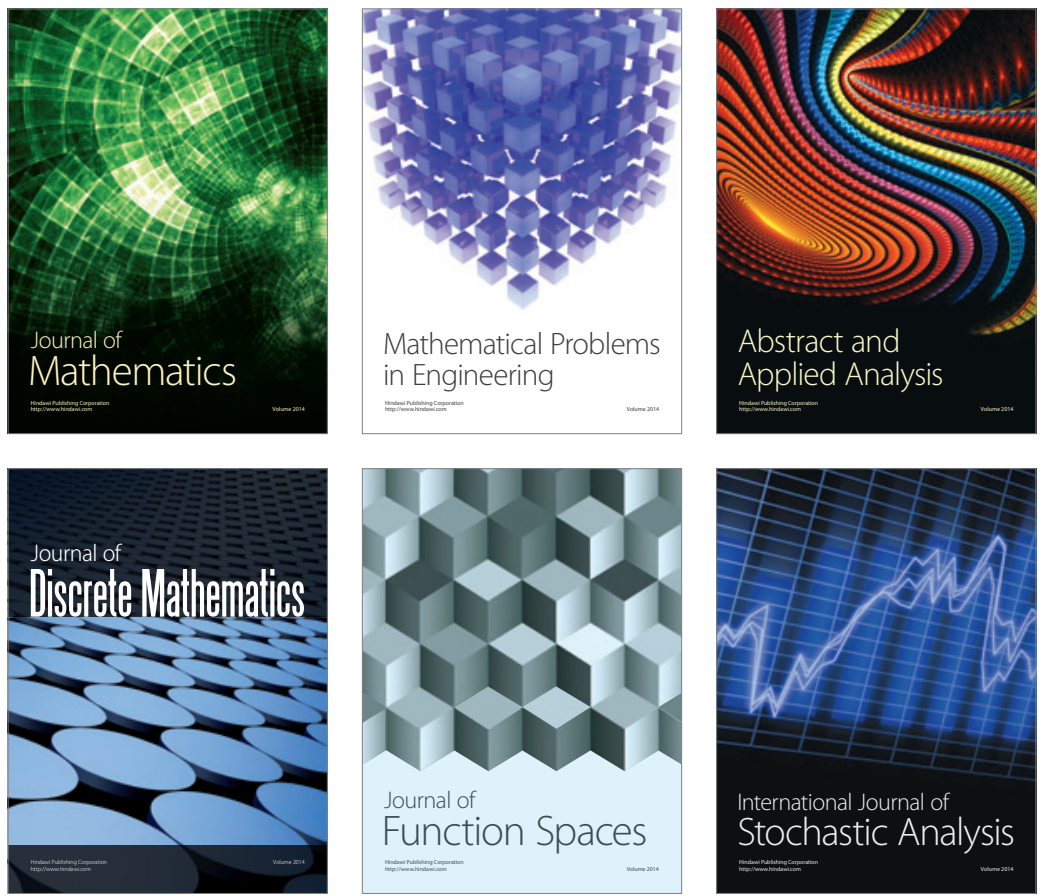

Journal of

Function Spaces

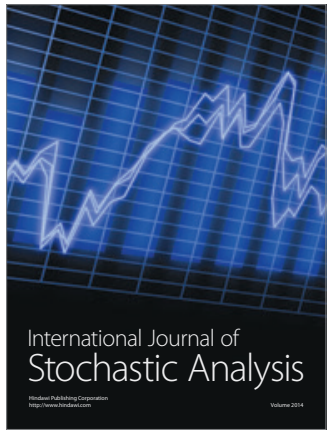

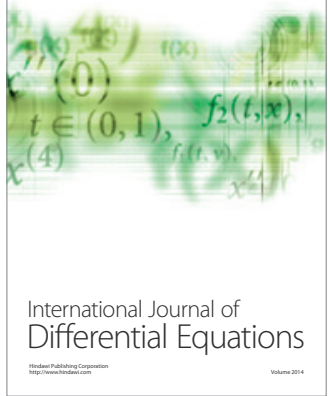
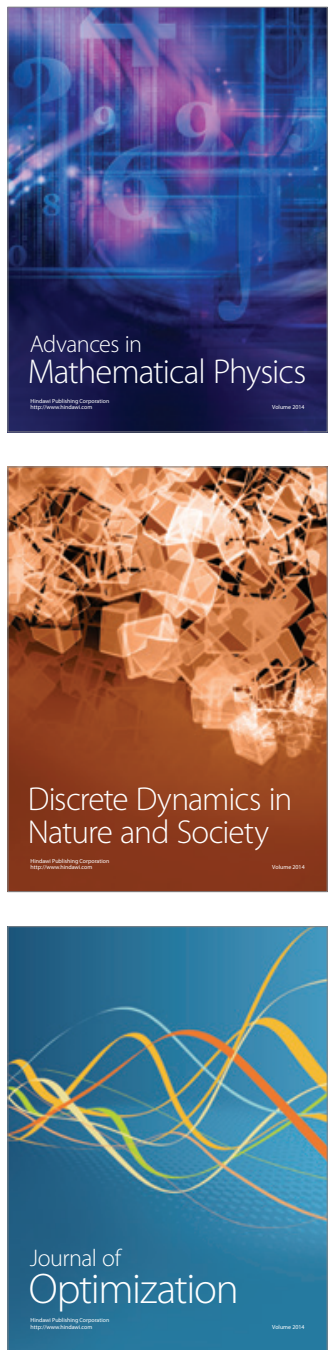